E3S Web of Conferences 1, 09006 (2013)

DOI: $10.1051 / \mathrm{e} 3$ sconf/20130109006

(c) Owned by the authors, published by EDP Sciences, 2013

\title{
Elimination of interferences from surface active substances in voltammetric determination of trace amounts of titanium in environmental water samples
}

\author{
M. Grabarczyk ${ }^{1}$ and M. Korolczuk ${ }^{1}$ \\ Faculty of Chemistry, Maria Curie-Sklodowska University, M. C. Sklodowska sq. 3, 20-031 Lublin, Poland \\ mgrabarc@poczta.umcs.lublin.pl,mkorolcz@poczta.umcs.lublin.pl
}

\begin{abstract}
A cathodic stripping voltammetric method for determination of Ti(IV) in water samples containing high concentrations of surfactants is described. The linear calibration plot for Ti(IV) was achieved in the simultaneous presence of $5 \mathrm{mg} \mathrm{L}^{-1}$ anionic, $1 \mathrm{mg} \mathrm{L}^{-1}$ cationic and $2 \mathrm{mg} \mathrm{L}^{-1}$ nonionic surfactants for an accumulation time of $30 \mathrm{~s}$ in the range $2.5 \times 10^{-8}$ to $5 \times 10^{-7} \mathrm{~mol} \mathrm{~L}^{-1}$, the detection limit for accumulation time of $30 \mathrm{~s}$ was about $8.4 \times 10^{-9} \mathrm{~mol} \mathrm{~L}^{-1}$. The developed method was successfully applied to Ti(IV) determination in environmental waters, such as river water (Bystrzyca, Czerniejówka), stagnant water (Lake Zemborzyce) and rain water (collected from eastern areas of Poland) with satisfactory results.
\end{abstract}

Key words: Titanium (IV), Determination, Adsorptive stripping voltammetry, Environmental waters

\section{Introduction}

The excellent properties possessed by titanium make it a highly useful material. Titanium alloys have been increasingly widely used in aviation, aerospace, shipbuilding and other industrial departments whereas the main use of $\mathrm{TiO}_{2}$ is as a white powder pigment used in products such as paints, coatings, plastics, paper, inks, fibers, food and cosmetics [Akram et al., 2011; Chunxiang et. A1., 2011]. Because application of titanium has been growing steadily, the possibility of rise of its concentration in environmental water samples increases. Therefore the need for simple, cheap and sensitive procedure of titanium determination in environmental water samples grows.

The electrochemical behavior of titanium makes its classical voltammetric determination very difficult, because at the common potentials it cannot be reduced to the metallic state and deposited onto an electrode. The voltammetric procedures for titanium determination are based on adsorption of the Ti(IV) complexes and next its reduction to Ti(III). For this purpose various complexing agents are exploited, e.g. mordant red, cupferron, beryllon III, pyrocatechol, calcein, xylenol orange, methylthymol blie, chromotripic acid, pyridylazoresorcinol.

The analysis of titanium at the trace level in various environmental samples has been achieved by adsorptive stripping voltammetric method which is based on the formation and accumulation of complexes with chloranilic acid.

However, it should be noted that although voltammetric methods are simple and sensitive, they are vulnerable to numerous interferences arising mainly from the environmental sample matrix. In voltammetric procedures even low concentrations of organic matter, inevitably present in environmental samples, limit the applicability, e.g. surfactants can foul and passive the electrode causing a decrease or total decay of the analytical signal [Hoyer and Jansen, 2003]. Because production and use of surfactants has been growing steadily, their concentration in environmental water samples increases [Chebotarev et al., 2004]. Therefore the need for voltammetric stripping procedures making possible the titanium determination in the presence of surfactants grows.

This work provides a simple and fast procedure for determination of Ti(IV), which allows for the analysis of environmental water samples with a complicated matrix containing high concentration of nonionic, anionic and cationic surfactants. For this purpose Amberlite XAD-7 resin was used for surface active substance removing by means of their adsorption on the resin while Ti(IV) remains in the sample and it is possible to directly determine it by adsorptive stripping voltammetry.

\section{Materials and Methods}


The measurements were made using an EA-9 electrochemical analyzer and a controlled growth static mercury drop electrode in the HMDE mode, both made by MTM-ANKO Cracow, Poland. A three electrode system consisting of an $\mathrm{Hg}$ drop working electrode (drop surface area $1.4 \mathrm{~mm}^{2}$ ), a Pt auxiliary electrode and an $\mathrm{Ag} / \mathrm{AgCl}$ reference electrode were used.

Measurements were performed using differential pulse adsorptive stripping voltammetry according to the following procedure. A natural water sample or synthetic sample for the testing procedure (containing Ti(IV) and surface active substances), $2 \mathrm{~mL}$ of $1 \mathrm{~mol} \mathrm{~L}^{-1}$ acetate buffer $\mathrm{pH}=3$ and an adequate volume of triply distilled water, so that the final volume of the solution was $20 \mathrm{~mL}$ were added to a glass vial of volume $25 \mathrm{~mL}$ and finally $0.5 \mathrm{~g}$ of XAD-7 resin was inserted. Then, a magnetic stirring bar was put into the vial, and the solution was mixed for $5 \mathrm{~min}$. Next, after sedimentation of resin 9.8 $\mathrm{mL}$ of the solution was pipetted into the electrochemical cell and then $200 \mu \mathrm{L}$ of $1 \times 10^{-2} \mathrm{~mol} \mathrm{~L}^{-1}$ chloranilic acid was added and deaeration for $5 \mathrm{~min}$. was performed. A mercury drop was formed, and the accumulation of the Ti(IV)-chloranilic acid complex was carried out at -0.25 $\mathrm{V}$ for $60 \mathrm{~s}$ from the stirred solution. After the equilibration time of $5 \mathrm{~s}$, the differential pulse voltammogram was recorded, while the potential was scanned from $-0.25 \mathrm{~V}$ to $-0.85 \mathrm{~V}$, with the intensity of the obtained peak directly proportional to the concentration of Ti(IV) in the sample. The scan rate and pulse height were $20 \mathrm{mV} \mathrm{s}^{-1}$ and $-50 \mathrm{mV}$, respectively.

\section{Results and Discussion}

\section{The elimination of influence of nonionic surface active compounds}

Triton X-100 is a high-purity, water-soluble, liquid nonionic surfactant that has come to be recognized as a performance standard among similar products. Triton $\mathrm{X}-100$ is widely used in numerous commercial and industrial products as a substrate for detergents, in textile and fiber manufacture because of its excellent detergency, excellent wetting ability and excellent grease and oil removal from hard surfaces [Mei-Hui, 2008]. Considering such a common utilization, Triton X-100 is released into the environment in relatively great amounts. Taking this into account Triton X-100 was chosen for the examination of the influence of nonionic surface active compounds on the voltammetric titanium signal and next elimination of these interferences using XAD-7 resin.

As can be observed, the voltammetric signal of titanium is very sensitive to the presence of even small amounts of the nonionic surfactant, $0.5 \mathrm{mg} \mathrm{L}^{-1}$ of Triton $\mathrm{X}-100$ causes almost total decay of the Ti(IV) peak. The addition of resin eliminates the unwanted negative influence of the nonionic surfactant. In the presence of Amberlite XAD-7 resin inherency of even $2 \mathrm{mg} \mathrm{L}^{-1}$ of Triton X-100 does not affect the titanium signal at all and $5 \mathrm{mg} \mathrm{L} \mathrm{L}^{-1}$ of Triton X-100 causes a decrease of the titanium signal to $60 \%$ of its original value.

The elimination of influence of cationic surface active compounds

Cationic surfactants are widely used in household products such as fabric softeners, hair conditioners, soaps, shampoo and other hair products. Other applications of cationic surfactants ensue from the fact that they are effective anti-bacteria agents, therefore, they are generally employed as disinfectants and antiseptic agents and are used in germicide and sanitizer products. Because of their positive charge, cationic surfactants adsorb strongly to the negatively charged surfaces of sludge, soil and sediments. The widespread use and sorption behavior of cationic surfactants implies that these substances are expected to be present in many environmental compartments [Agrawal et al., 2004]. One of them is a well-established commercial synthetic cationic surfactant cetyltrimethylammonium bromide (CTAB) and it was chosen for this work.

As can be observed, similarly as in the case of Triton X-100, the voltammetric signal of titanium is very sensitive to the presence of even small amounts of the cationic surfactant, $0.1 \mathrm{mg} \mathrm{L}^{-1}$ causes a decrease of the $\mathrm{Ti}(\mathrm{IV})$ peak to $60 \%$ of its original value and $0.5 \mathrm{mg} \mathrm{L}^{-1}$ causes total decay of the titanium signal.

The addition of Amberlite XAD-7 resin minimalize this negative influence. In the presence of resin inherence of even $2 \mathrm{mg} \mathrm{L}^{-1}$ of CTAB does not affect the titanium signal at all.

\section{The elimination of influence of anionic surface active compounds}

Anionic synthetic surfactant sodium dodecylsulfate (SDS) was selected for this work due to its high solubility in water and first of all because it is a well-established commercial anionic synthetic surfactant. SDS is commonly used as an ingredient in household and personal care products as well as in specialized applications [Mei-Hiu L, 2008].

SDS to a lesser extent influences the voltammetric signal of titanium, $5 \mathrm{mg} \mathrm{L}^{-1}$ causes a decrease of the peak to $60 \%$ of its original value. In the presence of Amberlite XAD-7 resin even $40 \mathrm{mg} \mathrm{L}^{-1}$ of SDS does not affect the titanium signal.

\section{Environmental samples analysis}

Four fresh environmental water samples, such as river water (Bystrzyca, Czerniejowka), stagnant water (Lake Zemborzyce) and rain water were collected from eastern areas of Poland and Ti(IV) determination by the proposed procedure was performed. No Ti(IV) was detected in the samples in concentrations above the detection limit. To confirm the accuracy of the proposed procedure the analysed samples were spiked with Ti(IV) at different concentration levels and the titanium content was determined by the standard addition method. Three replicate determinations using the standard addition method gave average recovery values between 97.3 and $102.8 \%$ with relative standard deviation between 4.8 and $2.6 \%$, what proves a satisfactory accuracy and precision of the proposed method for the determination of titanium 
in environmental water samples.

\section{Conclusion}

Analysis of titanium in natural water samples is of great relevance from the environmental point of view. In the literature data there are many voltammetric methods devoted to this case but the problems arise from the matrix of natural samples. The performed experiments demonstrate that the Amberlite XAD-7 resin can be effectively applied to eliminate interferences connected with the presence of surface active substances. The proposed procedure can be carried out directly in environmental water samples containing nonionic, anionic and cationic surfactants, without matrix removal. Additionally the method is simple, quick and requires no expensive equipment, which makes it available even for small laboratories.

\section{References}

Agrawal K, Agnihotri G, Shrivas K, Mundhara GL, Patel KS, Hoffmann P. Determination of Cationic
Surfactants in Environmental Samples by Flow Injection Analysis. Microchim Acta 2004; 147:273-278.

Akram M, Jansen KMB, Ernst LJ, Bhowmik S. Atmospheric pressure plasma surface modification of titanium for high temperature adhesive bonding. Inter J Adhes Adhes 2011; 31:598-604.

Chebotarev A N, Palenko TV, Shcherbakova TM. Adsorption-Photometric Determination of Cationic Surfactant Traces. J Anal Chem 2004; 59: 309-313.

Chunxiang C, BaoMin H, Lichen Z, Shuangjin L. Titanium alloy production technology, market prospects and industry development. Mater Design 2011; 32:1684-1691.

Hoyer B, Jansen N. Suppression of surfactant interferences in anodic stripping voltammetry by sodium dodecyl sulfate. Electrochem Commun 2003; 5:759-764.

Mei-Hiu L. Effects of nonionic and ionic surfactants on survival, oxidative stress, and cholinesterase activity of planarian . Chemosphere 2008; 70:1796-1803. 